\title{
Domain Decomposition for Heterogeneous Media
}

\author{
Ivan G. Graham ${ }^{1}$ and Patrick O. Lechner ${ }^{2}$ \\ 1 Department of Mathematical Sciences, University of Bath, Bath, BA2 7AY, \\ United Kingdom, igg@maths.bath.ac.uk, \\ 2 Department of Mathematical Sciences, University of Bath, Bath, BA2 7AY, \\ United Kingdom, mappol@maths.bath.ac.uk
}

Summary. Elliptic problems with multiscale coefficients have been studied to a great extent recently. Preconditioners based on standard domain decomposition methods often perform poorly when the variation of the coefficients inside the subdomains is large. In this paper we study the behaviour of domain decomposition methods based on linear coarsening for such problems and we also propose improved methods which use the notion of multiscale finite elements to define coarsening operators.

\section{Problem Description}

Typical examples of elliptic multiscale problems occur among others in fluid flow in strongly heterogeneous media or heat conduction in composite media. Let us therefore consider the second order partial differential equation of Poisson type

$$
-\nabla \cdot a(\mathbf{x}) \nabla u(\mathbf{x})=f(\mathbf{x}) \text { for } \mathbf{x} \in \Omega,
$$

with $\Omega \subset \mathbb{R}^{d}$, where $a(\mathbf{x})$ is the conductivity, which for simplicity is assumed to be scalar valued, symmetric and positive, but which is allowed to vary very strongly, typically as bad as $\max _{\mathbf{x}, \mathbf{y} \in \Omega}(a(\mathbf{x}) / a(\mathbf{y})) \cong 10^{9}$. Furthermore we assume $\Omega$ to be an interval for $d=1$, a polygon for $d=2$ and a polyhedron for $d=3$. We consider the Dirichlet problem with $u(\mathbf{x})=0$ for all $\mathbf{x} \in \partial \Omega$, the boundary of $\Omega$.

Closely related problems occur in the modeling of groundwater flow. Due to the difficulties in capturing the heterogeneity of rock formations and significant uncertainties away from the limited number of possible observation points, the permeability field, which is the (often strongly varying) multiscale field in this application, is then modeled stochastically, and in this case we consider a lognormal model $a(\mathbf{x}):=\exp (Z(\mathbf{x}))$, where $Z(\mathbf{x})$ is a Gaussian random field. Using Monte Carlo Methods on a large sample of reasonable 
realisations of these fields usually leads to good numerical results for $u$.

For physical and practical reasons (see [DN97]) fields $Z(\mathbf{x})$ of OrnsteinUhlenbeck type, i.e. statistically homogeneous isotropic Gaussian random fields with constant mean, variance $\sigma^{2}$, correlation length $\lambda$ and covariance function

$$
\Sigma(\mathbf{x}, \mathbf{y}):=\sigma^{2} \exp \{|\mathbf{x}-\mathbf{y}| / \lambda\}
$$

are considered.

We now discretise (1) using linear finite elements on a uniform triangulation of $\Omega$ with element diameter of order $h$, where the step size is in practice chosen so that $\lambda=C h$, for $C$ moderate, e.g. $C \approx 10$ (see Figure 1 ), to achieve an accuarate resolution of the problem without upscaling. Dark regions in these pictures represent areas with high permeability, etc.. The exact values depend on the variance $\sigma^{2}$. For large $\lambda$ the local variation of the field is reduced in general.

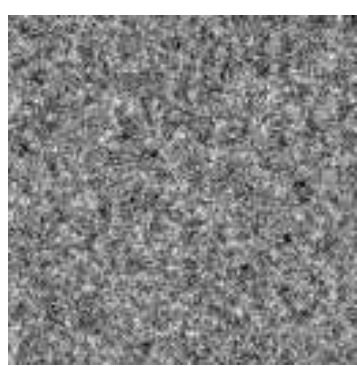

(a) Correlation length $\lambda=1 h$

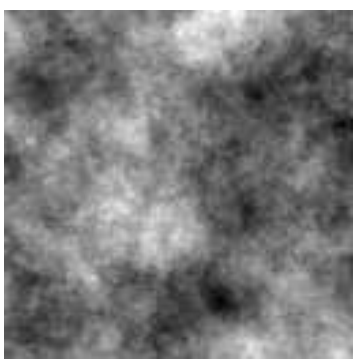

(c) Correlation length $\lambda=20 h$

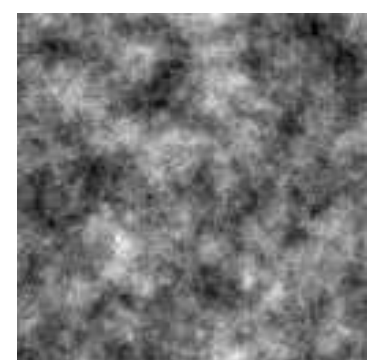

(b) Correlation length $\lambda=10 h$

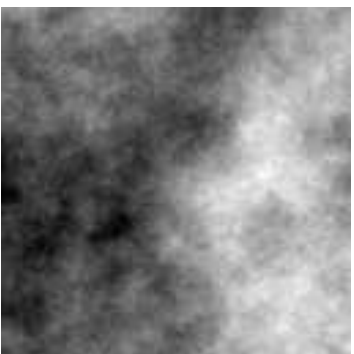

(d) Correlation length $\lambda=100 h$

Fig. 1: Dependence on the correlation length $\lambda$ on domain $[0,128 h]^{2}$

Now let $a$ be a fixed realisation of this random field and let $A(a)$ be the corresponding stiffness matrix with entries $A_{i j}(a):=\int_{\Omega} a \nabla \phi_{i} . \nabla \phi_{j}$, where $\left\{\phi_{i}\right\}$ 
are the piecewise linear nodal basis and let $A(1)$ be the stiffness matrix corresponding to a field with $a(\mathbf{x})=1$ for all $\mathbf{x} \in \Omega$. Then it can easily be shown that

$$
\kappa(A(a)) \leq \max _{\mathbf{x}, \mathbf{y} \subset \Omega}(a(\mathbf{x}) / a(\mathbf{y})) \cdot \kappa(A(1)),
$$

where $\kappa$ denotes the condition number.

Therefore the convergence of iterative methods, like the conjugate gradient method, depends on the global ratio of the coefficient $a$ and can be very slow for strongly varying $a$.

\section{Linear Interpolation Domain Decomposition}

The previous observations make it important to find a good preconditioner for the stiffness matrix $A(a)$ and we first of all study an additive Schwarz domain decomposition method with linear coarse space. We therefore introduce a coarse grid of size $H$, which defines triangular subdomains, $K_{i}$, that we extend to $p$ overlapping regions, $\hat{K}_{i}, i=1, \ldots, p$, with overlap $\delta$, such that the subdomains consist of unions of fine grid elements. Now let $R_{i}$ be the local restriction of a vector defined on the freedoms of the fine mesh to the freedoms on the interior of $\hat{K}_{i}$ and let $A_{i}:=R_{i} A R_{i}^{T}$. Also let $\left\{\mathbf{x}_{j}^{H}: j=1, \ldots, n_{c}\right\}$ be the set of coarse grid freedoms. Furthermore let $\left\{\phi_{i}^{H}\right\}$ be the set of linear interpolation functions with respect to the coarse grid, such that $\phi_{i}^{H}\left(\mathbf{x}_{j}^{H}\right)=\delta_{i j}$, where $\delta_{i j}$ denotes the Kronecker delta. Using these functions we introduce an interpolation map $R_{L}^{T}:=\left[\phi_{\mathbf{1}}^{\mathbf{H}}, \ldots, \boldsymbol{\phi}_{\mathbf{n}_{\mathbf{c}}}^{\mathbf{H}}\right]$, where for $i=1, \ldots, n_{c}, \phi_{\mathbf{i}}^{\mathbf{H}}$ is the vector of evaluations of $\phi_{i}^{H}$ at the fine grid freedoms. Finally set $A_{L}:=R_{L} A R_{L}^{T}$.

We can then show (using ideas discussed for example in [DW94], [CM94] and [TW04]), that for the two level linear interpolation additive Schwarz preconditioner $M_{L}^{-1}:=\sum_{i=0}^{p} R_{i}^{T} A_{i}^{-1} R_{i}+R_{L}^{T} A_{L}^{-1} R_{L}$, there exists a constant $C$, such that

$$
\kappa\left(M_{L}^{-1} A\right) \leq C \cdot B(d) \cdot \max _{i} \max _{\mathbf{x}, \mathbf{y} \subset K_{i}}(a(\mathbf{x}) / a(\mathbf{y}))\left(1+\delta^{-1} H\right),
$$

where $B(1)=1, B(2)=(1+\log (H / h))$ and $B(3)=H / h$.

When $a$ is moderately varying, better estimates which avoid the dependence on $H / h$ can be derived (see [TW04]). In general, for medium or large correlation length $\lambda$, the dependence on $a$ in (4) may be much better than in (3), since the subdomain ratios $\max _{i} \max _{\mathbf{x}, \mathbf{y} \subset K_{i}}(a(\mathbf{x}) / a(\mathbf{y}))$ can be much smaller than the global ratio $\max _{\mathbf{x}, \mathbf{y} \subset \Omega}(a(\mathbf{x}) / a(\mathbf{y}))$ (compare with Figure 1). Further details can be found in [Lec05]. 


\section{Multiscale Interpolation Domain Decomposition}

Estimate (4) still grows linearly with the global maximum ratio of permeability values over all subdomains. This can be improved by replacing the linear interpolation by a more suitable operator. For this we use ideas from multiscale finite elements (MsFE) (see [HW97] and [HWC99]). However we apply them as a way of improving solvers rather than improving accuracy. A similar motivation is discussed in [AH02]. The basic idea behind this is to use the fine scale structure of the problem in the construction of the coarse grid basis functions, which will then improve the coarse grid solves. For a typical triangular coarse grid element $K$ with nodes $\mathbf{x}_{j}^{K}, j=1,2,3$, we compute multiscale basis functions $\psi_{j}^{K}$ on the subdomain $K$ by solving the partial differential equations

$$
-\nabla \cdot a(\mathbf{x}) \nabla \psi_{j}^{K}(\mathbf{x})=0 \text { for } \mathbf{x} \in K
$$

where we force a Dirichlet boundary condition on $\psi_{j}^{K}$ with $\psi_{j}^{K}\left(\mathbf{x}_{i}^{H}\right)=\delta_{i j}$ and fix the behaviour of $\psi_{j}^{K}$ on the other parts of the boundary as discussed below. In practice $\psi_{j}^{K}$ is approximated by the finite element method on the fine mesh contained inside $K$.

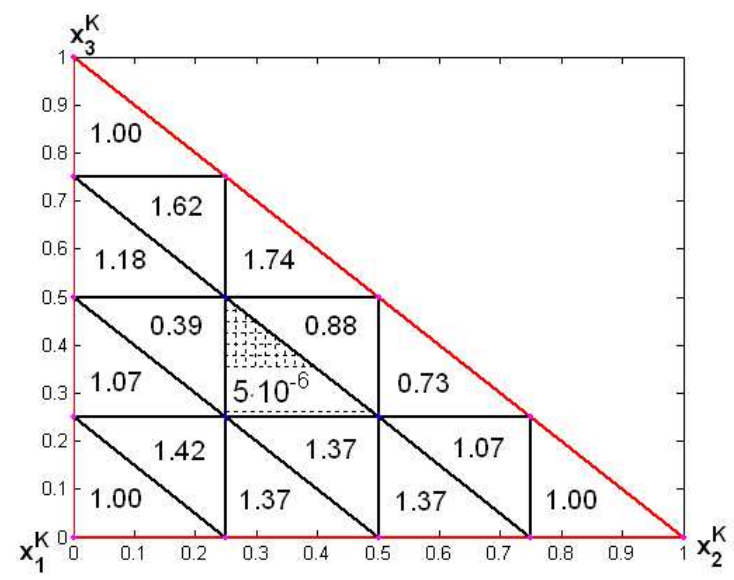

Fig. 2: Values of $H\left|\nabla \psi_{3}^{K}(\mathbf{x})\right|$ evaluated on the fine grid elements of subdomain $K$ for one multiscale basis function with $a=10^{6}$ on the marked element in the centre and $a=1$ everywhere else

In fine grid elements where the permeability is high the corresponding multiscale basis function will have a smaller gradient. This behaviour is illustrated in Figure 2, where $j=3$ and the permeability is taken as $a=10^{6}$ on one element and 1 on all the others. The $\psi_{j}^{K}$ are then combined to define nodal basis functions $\psi_{i}, i=1, \ldots, n_{c}$, which are defined on all of $\Omega$ with $\psi_{i}\left(\mathbf{x}_{j}^{H}\right)=\delta_{i j}$, 
$j=1, \ldots, n_{c}$, and $\left.\psi_{i}\right|_{K}$ is a linear combination of the $\psi_{l}^{K}, l=1,2,3$.

We then replace the linear interpolation map $R_{L}^{T}$ by a multiscale interpolation map $R_{M s}^{T}:=\left[\boldsymbol{\psi}_{\mathbf{1}}, \ldots, \boldsymbol{\psi}_{\mathbf{n}_{\mathbf{c}}}\right]$, where each $\boldsymbol{\psi}_{\mathbf{i}}$ is a vector of evaluations of $\psi_{i}$ at the fine grid nodes. The choice of the boundary condition in (5) can be very important. The simplest choice is to interpolate linearly between the the values $\psi_{j}\left(\mathbf{x}_{i}^{H}\right)=\delta_{i j}$ on the edges of $K$. Numerical results show, that these linear boundary conditions perform very well for small variance $\sigma^{2}$. However for large variance the performance can be improved by using so called oscillatory conditions as introduced in ([HW97], pages 172-173). Solving problems on extended subdomains, so called oversampling (useful for improving accuracy, see [HW97]), has not been found experimentally to improve the convergence rate significantly.

The new two level multiscale interpolation additive Schwarz preconditioner is now given by $M_{M s}^{-1}:=\sum_{i=0}^{p} R_{i}^{T} A_{i}^{-1} R_{i}+R_{M s}^{T} A_{M s}^{-1} R_{M s}$.

We compare the performance of this preconditioner with that of a standard linear two-level additive Schwarz preconditioner. Consider therefore a simple two-dimensional problem on $[0,1]^{2}$ on which we define a uniform triangular fine mesh of size $h$ and a coarse triangular mesh of size $H$ and fix a constant (minimal) overlap $\delta=2 h$. The following tables then compare the iteration numbers and computation times (in brackets; including setup time and iteration time) of the two preconditioners for $h=1 / 128$ and $H=8 h$, resp. $H=16 h$ and for different variances $\sigma^{2}$, where in the case of multiscale interpolation, oscillatory boundary conditions were used for the construction of the interpolation functions. Here Table 1 is for fields of Ornstein-Uhlenbeck type with correlation length $\lambda=10 h$ and Table 2 for completely random isotropic fields. Both tables show, that in two dimensions especially for strongly varying fields the multiscale interpolation brings a considerable improvement in both iteration numbers and computation times.

\begin{tabular}{||c||rr|rr||rr|rr||}
\hline \hline \multicolumn{1}{||c||}{\begin{tabular}{||cr||rr||} 
Variance \\
$\sigma^{\mathbf{2}}$
\end{tabular}} & \multicolumn{4}{c||}{$\mathbf{H}=\mathbf{8 h}$} & \multicolumn{4}{c||}{$\mathbf{H}=\mathbf{1 6 h}$} \\
\hline 1 & 29 & $(90)$ & 27 & $(89)$ & 43 & $(175)$ & 42 & $(178)$ \\
2 & 33 & $(100)$ & 29 & $(93)$ & 45 & $(180)$ & 43 & $(180)$ \\
4 & 41 & $(118)$ & 34 & $(101)$ & 53 & $(199)$ & 49 & $(192)$ \\
8 & 59 & $(160)$ & 45 & $(127)$ & 73 & $(239)$ & 62 & $(214)$ \\
16 & 109 & $(277)$ & 70 & $(184)$ & 144 & $(388)$ & 90 & $(269)$ \\
24 & 187 & $(455)$ & 96 & $(245)$ & 251 & $(611)$ & 122 & $(342)$ \\
\hline \hline
\end{tabular}

Table 1: Iteration numbers and computation times (in sec.) for fields of Ornstein-Uhlenbeck type with $\lambda=10 h$ 


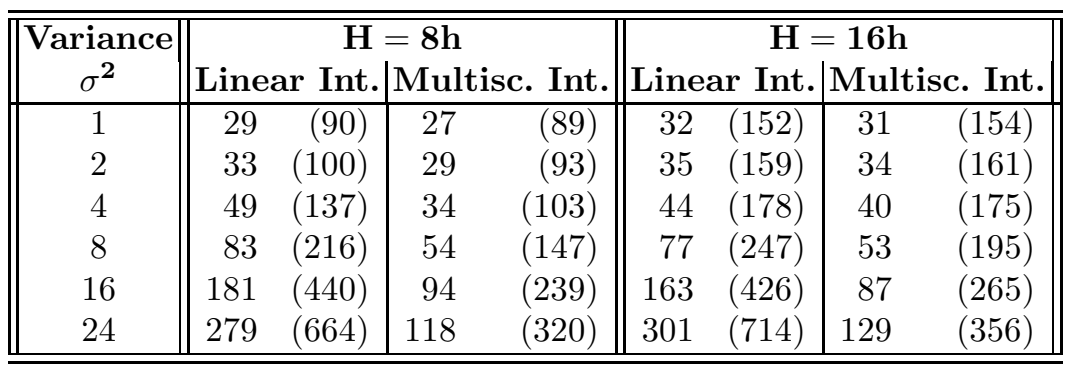

Table 2: Iteration numbers and computation times (in sec.) for completely random isotropic fields

For one dimensional problems, one can show, that $M_{M s}^{-1}$ is in fact the exact inverse of $A$, when the subdomains have zero overlap. The same is true in higher dimensions, if we replace the node based version of the multiscale preconditioner by a skeleton based version, which means we compute a multiscale basis function $\psi_{i}$ for every single freedom of the skeleton (i.e. each fine grid freedom on the boundary of the subdomains), instead of only using the basis functions corresponding to the coarse grid freedoms. Multiscale preconditioners with complexity between the node based and skeleton based methods and their performance are part of our current research and will be discussed in [Lec05].

\section{References}

[AH02] Aarnes, J., Hou, T.Y.: Multiscale Domain Decomposition Methods for Elliptic Problems with High Aspect Ratios. Acta Mathematicae Applicatae Sinica, English Series, 18, No. 1, 63-76 (2002)

[CM94] Chan, T.F., Mathew, T.P.: Domain Decomposition Survey. In: Iserles, A. (ed), Acta Numerica 1994, 61-143. Cambridge University Press, Cambridge (1994)

[DN97] Dagan, G., Neumann, S.P.: Subsurface Flow and Transport: A Stochastic Approach. Cambridge University Press, Cambridge (1997)

[DW94] Dryja, M., Widlund, O.B.: Domain decomposition algorithms with small overlap, 604-620. SIAM J. Sci. Comp., 15(3) (1994)

[HW97] Hou, T.Y., Wu, X.H.: A Multiscale Finite Element Method for Elliptic Problems in Composite Materials and Porous Media. J. Comp. Phys., 134, 169-189 (1997)

[HWC99] Hou, T.Y., Wu, X.H., Cai, Z.: Convergence of a multiscale finite element method for elliptic problems with rapidly oscillating coefficients. Math. Comp., 68, 913-943 (1999)

[Lec05] Lechner, P.O.: PhD thesis, in preparation, University of Bath (2005)

[TW04] Toselli, A., Widlund, O.: Domain decomposition methods - algorithms and theory. Springer, Berlin (2004) 